\title{
Electromagnetic signature of supermassive black hole binaries that enter their gravitational-wave induced inspiral
}

\section{Citation}

Loeb, Abraham. 2010. "Electromagnetic Signature of Supermassive Black Hole Binaries That Enter Their Gravitational-Wave Induced Inspiral." Physical Review D 81 (4). https:// doi.org/10.1103/physrevd.81.047503.

\section{Permanent link}

http://nrs.harvard.edu/urn-3:HUL.InstRepos:41412250

\section{Terms of Use}

This article was downloaded from Harvard University's DASH repository, and is made available under the terms and conditions applicable to Open Access Policy Articles, as set forth at http:// nrs.harvard.edu/urn-3:HUL.InstRepos:dash.current.terms-of-use\#OAP

\section{Share Your Story}

The Harvard community has made this article openly available. Please share how this access benefits you. Submit a story. 


\title{
An Electromagnetic Signature of Supermassive Black Hole Binaries That Enter Their Gravitational-Wave Induced Inspiral
}

\author{
Abraham Loeb \\ Astronomy Department, Harvard University, 60 Garden St., Cambridge, MA 02138, USA
}

(Dated: November 14, 2018)

\begin{abstract}
Mergers of gas-rich galaxies lead to black hole binaries that coalesce as a result of dynamical friction on the ambient gas. Once the binary tightens to $\lesssim 10^{3}$ Schwarzschild radii, its merger is driven by the emission of gravitational waves (GWs). We show that this transition occurs generically at orbital periods of $\sim 1-10$ years and an orbital velocity $v$ of a few thousand $\mathrm{km} \mathrm{s}^{-1}$, with a very weak dependence on the supply rate of gas $\left(v \propto \dot{M}^{1 / 8}\right)$. Therefore, as binaries enter their GWdominated inspiral, they inevitably induce large periodic shifts in the broad emission lines of any associated quasar(s). The probability of finding a binary in tighter configurations scales as $v^{-8}$ owing to their much shorter lifetimes. Narrow-band monitoring of the broad emission lines of quasars on timescales of months to decades can set a lower limit on the expected rate of GW sources for LISA.
\end{abstract}

PACS numbers: $04.25 . \mathrm{dg}, 98.62 . \mathrm{Js}, 98.54 . \mathrm{Aj}, 98.62 . \mathrm{Ra}$

Introduction. Recent advances in general relativistic simulations of the final coalescence phase of black hole $(\mathrm{BH})$ binaries through gravitational wave (GW) emission [1], sparked new interest in related electromagnetic (EM) signals 2]. New observational searches were initiated for wide $\mathrm{BH}$ binaries in their pre-merger phase or single quasars which are displaced from their host galaxy spatially or spectroscopically due to GW recoil after the merger [3]. In addition to testing the recent numerical predictions, such searches can be used to calibrate the expected rate of GW sources for the proposed LISA mission [4], as well as to reduce the error box on the source localization after LISA is launched. The next generation of simulations will attempt to incorporate gas dynamics and radiative transfer in order to calculate the EM counterparts to GW sources [5]. If these EM signatures are sufficiently unusual relative to typical quasar variability, one could search for them well before LISA is launched. Since BH binaries spend more time at larger separations, wider binaries should be more abundant in quasar surveys. In this paper we study a generic EM signature of the widest quasar binaries whose dynamics is dominated by $\mathrm{GW}$ emission.

The formation of $\mathrm{BH}$ binaries is a natural by product of galaxy mergers (which are generic to the hierarchical build-up of galaxies in the standard cosmological model), since almost all galaxies in the local Universe are observed to harbor a $\mathrm{BH}$ at their center [6]. The tidal torques generated during a merger extract angular momentum from any associated cold gas and concentrate the gas near the center of the merger remnant, where its accretion onto a BH results in a bright quasar 7].

Binary Parameters and Statistics. We consider two black holes with masses $M_{1}$ and $M_{2}$ in a circular orbit of radius $a$ about each other [8]. Their respective distances from the center of mass are $a_{i}=\left(\mu / M_{i}\right) a(i=1,2)$, where $\mu=M_{1} M_{2} / M$ and $M=M_{1}+M_{2}$. We define the parameter $\zeta=4 \mu / M$, which equals unity if $M_{1}=M_{2}$ and is smaller otherwise. The orbital period is given by,

$$
P=2 \pi\left(G M / a^{3}\right)^{-1 / 2}=1.72 \operatorname{yr} a_{16}^{3 / 2} M_{8}^{-1 / 2},
$$

where, $a_{16} \equiv\left(a / 10^{16} \mathrm{~cm}\right)$ and $M_{8} \equiv\left(M / 10^{8} M_{\odot}\right)$. The angular momentum of the binary can be expressed in terms of the absolute values of the velocities of its members $v_{1}$ and $v_{2}$ as $J=\Sigma_{i=1,2} M_{i} v_{i} a_{i}=\mu v a$, where the relative orbital speed is

$$
v=v_{1}+v_{2}=(2 \pi a / P)=1.15 \times 10^{4} \mathrm{~km} \mathrm{~s}^{-1} M_{8}^{1 / 2} a_{16}^{-1 / 2} .
$$

We focus our discussion on mergers of gas-rich galaxies since those are most likely to result in bright quasars which are easy to observe. Hydrodynamic simulations of such mergers indicate that the associated $\mathrm{BH}$ binaries inspiral steadily as a result of dynamical friction on the ambient gas [9]. In contrast, $\mathrm{BH}$ binaries in gas-poor mergers, which shrink by scattering stars, may stall once they reach a separation $a \lesssim 1 \mathrm{pc}$ if the supply time of new stars which cross their orbit exceeds the Hubble time (resulting in the so-called "final parsec problem" [10]). In gas-rich mergers, the rate of inspiral slows down as soon as the gas mass interior to the binary orbit is smaller than $\mu$ and the enclosed gas mass is no longer sufficient for carrying away the entire orbital angular momentum of the binary, $J$ [11]. Subsequently, momentum conservation requires that fresh gas will steadily flow towards the binary orbit in order for it to shrink. The binary tightens by expelling gas out of a region twice as large as its orbit (similarly to a "blender" opening a hollow gap [12]) and by torquing the surrounding disk through spiral arms 13, 14, 15]. Fresh gas re-enters the region of the binary as a result of turbulent transport of angular momentum in the surrounding disk. Since the expelled gas carries a specific angular momentum of $\sim v a$, the coalescence time of the binary is inversely proportional to the supply rate of fresh gas into the binary region. In a steady state, the mass supply rate of gas that extracts angular 
momentum from the binary, $\dot{M}$, is proportional to the accretion rate of the surrounding gas disk. Given that a fraction of the mass that enters the central gap accretes onto the BHs and fuels quasar activity [16, 17], it is appropriate to express $\dot{M}$ in Eddington units $\dot{\mathcal{M}} \equiv \dot{M} / \dot{M}_{E}$, corresponding to the accretion rate required to power the limiting Eddington luminosity with a radiative efficiency of $10 \%, \dot{M}_{E}=2.3 M_{\odot} \mathrm{yr}^{-1} M_{8}$. We then find,

$$
t_{\text {gas }} \approx(J / \dot{M} v a)=\mu / \dot{M}=1.1 \times 10^{7} \text { yr } \zeta \dot{\mathcal{M}}^{-1} .
$$

For a steady $\dot{\mathcal{M}}$, the binary spends equal amounts of time per $\log a$ until GWs start to dominate its loss of angular momentum.

The coalescence timescale due to GW emission is given by [18],

$$
t_{\mathrm{GW}}=\frac{5}{256} \frac{c^{5} a^{4}}{G^{3} M^{2} \mu}=2.53 \times 10^{5} \text { yr } \frac{a_{16}^{4}}{\zeta M_{8}^{3}} .
$$

By setting $t_{\mathrm{GW}}=t_{\text {gas }}$ we can solve for the orbital speed, period, and separation at which GWs take over,

$$
\begin{aligned}
v_{\mathrm{GW}} & =7.2 \times 10^{3} \mathrm{~km} \mathrm{~s}^{-1} \zeta^{-1 / 4}\left(\dot{\mathcal{M}} M_{8}\right)^{1 / 8} ; \\
P_{\mathrm{GW}} & =7.1 \mathrm{yr} \zeta^{3 / 4} M_{8}^{5 / 8} \dot{\mathcal{M}}^{-3 / 8} ; \\
a_{\mathrm{GW}} & =8.3 \times 10^{-3} \mathrm{pc} \zeta^{1 / 2} M_{8}^{3 / 4} \dot{\mathcal{M}}^{-1 / 4} .
\end{aligned}
$$

For a binary redshift $z$, the observed period is $(1+z) P_{\mathrm{GW}}$. Remarkably, the orbital speed at which GWs take over is very weakly dependent on the supply rate of gas, $v_{\mathrm{GW}} \propto$ $\dot{M}^{1 / 8}$. It generically corresponds to an orbital separation of order $\sim 10^{3}$ Schwarzschild radii $\left(2 G M / c^{2}\right)$.

In an equal-mass binary, each $\mathrm{BH}$ moves relative to the center-of-mass at a speed of $\frac{1}{2} v_{\mathrm{GW}}$. Coincidentally, this orbital speed happens to be similar to the velocity width of the broad emission lines (BEL) of quasars [19]. This fortunate coincidence implies that the timedependent gravitational potential and UV illumination of the binary members will introduce variability into the spectroscopic shape of any associated BEL. It is believed that BEL are emitted by numerous compact clouds which cover about a tenth of the sky of the quasar [19]. Even if the BEL clouds originate in the outer edge of the accretion disk around one of the BHs, their dynamics will be affected by the combined gravitational force and UV illumination from both BHs.

We therefore robustly predict that when $B H$ binaries from mergers of comparable-mass, gas-rich galaxies start to inspiral by $G W$ emission, they would show variability with a period of months to decades (for $M(1+z)^{8 / 5} \sim$ $\left.10^{6}-10^{9} M_{\odot}\right)$ in any associated BEL, across a range of Doppler velocities comparable to the BEL spectral width.

The notion that $\mathrm{BH}$ binaries would display periodic shifts in their emission lines was recognized a long time ago [20]. So far, intensive monitoring programs for a limited number of active galactic nuclei revealed spectroscopic variability but no reproducible evidence for a periodic signal 21]. The new insight added by Eqs. (5+7) is that $\mathrm{BH}$ binaries should generically start their GWinduced inspiral at orbital periods that are observationally accessible, and at orbital velocities that are comparable to the BEL width. This would naturally lead to a double-peaked line profile which can be observed to vary periodically. In contrast, the line profile is expected to remain singly peaked [38] at the lower orbital velocities associated with wider binary separations (where the superposed BEL of the two BHs are only slightly offset in velocity relative to each other), or for binaries that are much more compact than their common BEL region (and hence act gravitationally as a single $\mathrm{BH}$ ).

The periodic Doppler shift of the BEL is expected to be smaller for unequal mass binaries. If each $\mathrm{BH}$ controls the spectroscopic centroid of its own BEL feature, the BEL associated with $M_{i}$ will be shifted by $v_{i}=\left(\mu / M_{i}\right) v$. The relative abundance of $\mathrm{BH}$ binaries with different mass ratios can be modeled theoretically [22].

The probability of observing periodic BEL shifts due to a binary transitioning to a GW-dominated inspiral, $\mathcal{P}_{\mathrm{GW}}$, depends on the unknown fraction of the associated quasar lifetime which overlaps with this final phase of binary coalescence. In case accretion is suppressed by the opening of an inner cavity in the disk [12], it is possible that no BEL emission is observable early in the GW-dominated inspiral phase. However, recent numerical simulations 13, 14, 15, 16, 17] indicate that accretion should continue during this phase through the formation of two secondary accretion disks around the individual BHs. If quasar emission persists throughout the coalescence process at a constant $\dot{\mathcal{M}}$ due to the abundant supply of cold galactic gas, then one is likely to find as many binaries with separation $a_{\mathrm{GW}} \sim 10^{-2} \mathrm{pc}$ as with a separation of tens of pc (for which the enclosed gas mass is comparable to $\mu$ ). In that case, the prospects for discovering a quasar binary with a periodic BEL shift are good, since an example for a tight binary at a projected separation of $\sim 7.3 \mathrm{pc}$ has already been identified [23] and three sub-pc candidates have been reported recently [24].

The probability of finding binaries deeper in the GWdominated regime, $\mathcal{P} \propto t_{\mathrm{GW}}$, diminishes rapidly at increasing orbital speeds, with $\mathcal{P}=\mathcal{P}_{\mathrm{GW}}\left(v / v_{\mathrm{GW}}\right)^{-8}$.

Discussion. State-of-the-art numerical simulations [15, 17] focused so far on wide binary separations where gravitational torques dominate the interaction of the binary with the surrounding gas, but did not attempt to describe the late, viscosity-driven, evolutionary phase of interest here. This late inspiral phase is mediated by angular momentum transport in the circumbinary disk which delivers fresh gas from large distances into the binary region. Since quasar accretion disks on scales of $\sim 10^{4}$ Schwarzschild radii are radiation-pressure dominated, a proper numerical treatment of the turbulent transport of angular momentum requires a radiationmagneto-hydrodynamics code. Suitable numerical sim- 
ulations were only applied to a single BH so far [25], but could be extended with a global code 26] to the binary accretion problem.

Given the limitations of existing simulations, one is restricted to modeling the inflow of gas into the binary orbit through analytic approximations. A recent analytic treatment of viscous transport within a circumbinary accretion disk 11] ignored the existence of individual compact accretion disks around the two BHs and adopted a simplified prescription for $\alpha$-viscosity and binary migration. The formation of two compact accretion disks around the BHs (that is necessary for producing BEL) is expected to occur through gas inflow from the inner edge of the circumbinary disk [27], and the moving inner disks could affect the gas flow around the binary both hydrodynamically [16] and radiatively (especially if they radiate near the Eddington limit [17]). Bearing these simplifications in mind, Fig. 3 in Ref. [11] is in excellent agreement with Eq. (5), confirming that the GW-driven phase is approached at a characteristic orbital velocity of $\sim 10^{4} \mathrm{~km} \mathrm{~s}^{-1}$ with only a weak dependence on the accretion model details. The generic results of Eqs. (5-7) naturally lead to double-peaked line profiles which vary periodically on timescales of months to decades.

Future monitoring of the broad emission lines of quasars can set a lower limit on the expected rate of GW sources for LISA. A multi-year search dedicated to periodic variability of BEL in a large sample of quasars has not been performed as of yet. Preliminary studies have already made use of spectroscopic data from SDSS 28. and SDSS-III 29]. Any related discovery would motivate the implementation of narrow-band filters in photometric variability surveys, culminating with the future LSST [30]. A precursor for this approach may be realized in the near future.

The Palomar Transient Factory [31] (PTF) plans to acquire $\mathrm{H} \alpha$ filters at $6564 \AA$ which are $80-100 \AA$ wide. The periodic Doppler shift of the BEL in quasar binaries that enter their GW-dominated inspiral corresponds to a fractional wavelength change of $\left(v_{\mathrm{GW}} / c\right) \sim 1 \%$ or $\sim 66 \AA$. It would be possible to notice such a shift as an observed line slides in and out of the above mentioned filters. The sky surface density of quasars brighter than an i-band magnitude of 19 , for which the $\mathrm{Mg}$ II, C IV or C III lines are redshifted to $6564 \AA$, is $\sim 0.2$ per square degree on the sky. This results in 3 quasars per pointing if two filters are used. The planned survey will monitor up to several hundred fields containing up to $\sim 10^{3}$ quasars with a cadence of 1 month, and measure relative i-band fluxes to a precision of $0.3 \%$ (3 milli-magnitudes). It is also possible to design a dedicated survey that will cover $40 \%$ of the sky (a few thousand quasars) with a 1-year cadence. Separately from this program, a survey is underway to study variability (reverberation mapping) of quasars brighter than an r-band magnitude of 21 using six $200 \AA$ wide filters of the COMBO-17 survey [32] on the Chandra Deep Field South, using the ESO $2.2 \mathrm{~m}$ telescope at La Silla [33].

Spectroscopic surveys are much more labor intensive. Previous spectroscopic searches for BEL variability did not report any detection of periodicity for several tens of quasars that were monitored over many years [34] or in a sample of a few thousand quasars that were sampled only a few times over less than a year [35, 36], but identified examples of longer-term variability without a measured period [20].

Since quasar BEL are known to vary for other reasons [21], it is crucial to identify periodicity before classifying a candidate source as real. In confirmed cases, measurements of the period and velocity shift can be used to solve for $M$ and $a$ up to an ambiguity regarding the inclination angle of the orbital plane relative to the line of sight. This ambiguity may be removed through reverberation mapping [19, 36], since a measurement of the time delays between temporal variations in the UV continuum originating near the $\mathrm{BHs}$ and the two response times of their BEL, would provide an independent estimate of $M_{1}$ and $M_{2}$ (and hence $\zeta$ ). Even before periodic variability is observed, reverberation studies can be used to distinguish the double line profile of a binary (for which the two lines would respond differently to variations in the UV continuum of the two quasars [37]) from that of a single disk emitter (for which the two lines will vary simultaneously). In addition, the quasar luminosity could be used to estimate $\dot{M}$ based on related hydrodynamic simulations [13, 14, 15, 16, 17]. With all this information, observers will be in a position to examine whether $t_{\text {gas }}$ is indeed of order $t_{\mathrm{GW}}$ in these $\mathrm{BH}$ binaries.

Acknowledgments. I thank Y. Birnboim, L. Blecha, B. Kocsis, E. Ofek, and N. Stone for a careful reading of the manuscript and helpful comments. I also thank the Joint Space science Institute at the Univ. of Maryland for organizing a workshop that inspired this paper. This work was supported in part by NSF grant AST-0907890.

[1] F. Pretorius, Phys. Rev. Lett. 95, 121101 (2005); J. G. Baker, J. Centrella, D. I. Choi, M. Koppitz, \& J. van Meter, Phys. Rev. Lett. 96, 111102 (2006); M. Campanelli, C. O. Lousto, P. Marronetti, \& Y. Zlochower, Phys. Rev. Lett. 96, 111101 (2006).

[2] A. Loeb, Phys. Rev. Lett. 99, 041103 (2007); G. A. Shields, \& E. W. Bonning, Astrophys. J. 682, 758 (2008); B. Kocsis, \& A. Loeb, Phys. Rev. Lett. 101, 041101 (2008); L. Blecha, \& A. Loeb, Mon. Not. R. Astr. Soc. 390, 1311 (2008); Z. Lippai, Z. Frei, \& Z. Haiman, Astrophys. J. 676, L5 (2008); J. D. Schnittman, \& J. H. Krolik, Astrophys. J. 684, 835 (2008); T. Bogdanović, et al., Astrophys. J. Suppl. 174, 455 (2008); S. Komossa, \& D. Merritt, Astrophys. J. 683, L21 (2008), ibid, 689, L89 (2008); R. Mohayaee, et al., Astrophys. J. Lett. 674, 21 (2008); R. O'Leary, \& A. Loeb, Mon. Not. R. As- 
tron. Soc. 395, 781 (2009); D. Merritt, J. D. Schnittman, \& S. Komossa, Astrophys. J. 699, 1690 (2009); S. M. O'Neill, et al., Astrophys. J. 700, 859 (2009); B. Devecchi, et al., Mon Not. R. Astron. Soc. 394, 633 (2009); Z. Haiman, et al., Class. Quant. Grav. 26, 094032 (2009); P. Chang, et al., Mon. Not. R. Astron. Soc., submitted (2009) arXiv:0906.0825.

[3] E. W. Bonning, G. A. Shields, \& S. Salviander, Astrophys. J. 666, L13 (2007); G. A. Shields, \& E. W. Bonning, Astrophys. J. 682, 758 (2008); S. Komossa, et al., Astrophys. J. 678, L81 (2008); J. M. Comerford, et al., Astrophys. J. 698, 956 (2009); ibid 702, L82 (2009); X. Lin, et al., Astrophys. J., submitted (2009) arXiv:0908.2426; K. Smith, et al., Astrophys. J., submitted (2009) arXiv:0908.1998; G. A. Shields, et al., Astrophys. J., submitted (2009) arXiv:0907.3470. See also, S. Komossa, et al., Astrophys. J. 582, L15 (2003); H. Zhou, et al., Astrophys. J. 604, 33 (2004); S. Komossa, Mem. d. Soc. Astron. Ital. 77, 733 (2006), B. F. Gerke, et al., Astrophys. J. Lett. 660, 23 (2007).

[4] http://lisa.nasa.gov/

[5] J. R. van Meter, et al., arXiv:0908.0023 (2009); C. Palenzuela, et al., Phys. Rev. Lett. 103, 081101 (2009).

[6] K. Gültekin, et al., Astrophys. J. 698, 198 (2009), and references therein.

[7] A. Toomre, \& J. Toomre, Astrophys. J. 178, 623 (1972); J. E. Barnes, \& L. Hernquist, Ann. Rev. Astron. \& Astrophys. 30, 705 (1992); T. Di Matteo, V. Springel, \& L. Hernquist, Nature 433, 604 (2005).

[8] Orbital eccentricity is damped by dynamical friction on the ambient gas prior to the compact phase considered in this paper; see P. J. Armitage, \& P. Natarajan, Astrophys. J. 634, 921 (2005).

[9] M. Colpi, \& M. Dotti, arXiv:0906.4339 (2009), and references therein; A. Escala, et al., Astrophys. J. 630, 152 (2005); L. Mayer, et al., Science 316, 1874 (2007); S. Callegari, et al., Astrophys. J. 696, L89 (2009).

[10] M. C. Begelman, R. D. Blandford, \& M. J. Rees, Nature 287, 307 (1980); D. Merritt, \& M. Milosavljević, Liv. Rev. Rel. 8, 8 (2005), and references therein; P. Berczik, et al., Astrophys. J. 642, L21 (2006); A. Sesana, et al., Astrophys. J. 660, 546 (2007); H. Perets, et al., Astrophys. J. 656, 709 (2007).

[11] Z. Haiman, B. Kocsis, \& K. Menou, Astrophys. J. 700, 1952 (2009). Earlier analytic estimates on related problems were made by P. Artymowicz, \& S. H. Lubow, Astrophys. J. 421, 651 (1994); D. Syer, \& C. J. Clarke, Mon. Not. R. Astron. Soc. 277, 758 (1995); P. B. Ivanov, J. C. B. Papaloizou, \& A. C. Polnarev, Mon. Not. R. Astron. Soc. 307, 79 (1999); A. Gould, \& H.-W. Rix, Astrophys. J. 532, L29 (2000); and P. J. Armitage, \& P. Natarajan, Astrophys. J. 567, L9 (2002).

[12] M. Milosavljević, \& E. S. Phinney, Astrophys. J. 622, 93 (2005).

[13] K. Hayasaki, S. Mineshige, \& H. Sudou, Pub. Astron. Soc. Jap. 59, 427 (2007).

[14] A. I. MacFadyen, \& M. Milosavljević, Astrophys. J. 672, 83 (2008).

[15] J. Cuadra, et al., Mon. Not. R. Astron. Soc. 393, 1423 (2009).

[16] K. Hayasaki, Pub. Astron. Soc. Jap. 61, 65, (2009); K. Hayasaki, S. Mineshige, S., \& L. C. Ho, Astrophys. J.
682, 1134 (2008)

[17] M. Dotti, et al., Mon. Not. R. Astron. Soc. 396, 1640 (2009).

[18] P. C. Peters, Phys. Rev. 136, 1224 (1964); S. L. Shapiro \& S. A. Teukolsky, Black Holes, White Dwarfs and Neutron Stars, New-York: Wiley (1983), p. 477. The expression for a circular orbit was first derived by A. Einstein, Sitzungsberichte der Königlich Preußischen Akademie der Wissenschaften (Berlin), p. 154 (1918), and corrected by A. Eddington, Proc. R. Soc. London 102, 268 (1922).

[19] B. Peterson, An Introduction to Active Galactic Nuclei, Cambridge: Cambridge Univ. Press (1997), pp. 67-93.

[20] C. M. Gaskell, Liege International Astrophysical Colloquia 24, 473 (1983); Astrophys. J. 464, L107 (1996). S. G. Sergeev, et al., Astrophys. J. 668, 708 (2007); S. Gezari, J. P. Halpern, \& M. Eracleous, Astrophys. J. Suppl. 169, 167 (2007).

[21] J. P. Halpern, \& A. Filippenko, Nature 331, 46 (1988); AIP Conf. Ser. 254, 57 (1992); S. G. Sergeev, et al., Astrophys. J. 668, 708 (2007); S. Gezari, J. P. Halpern, \& M. Eracleous, Astrophys. J. Suppl. 169, 167 (2007).

[22] M. Volonteri, F. Haardt, \& P. Madau, Astrophys. J. 582, 559 (2003); A. Sesana, M. Volonteri, \& F. Haardt, Class. Quant. Grav. 26, 094033 (2009), and references therein.

[23] C. Rodriguez, et al., Astrophys. J. 697, 37 (2009); ibid 646, 49 (2006).

[24] T. Bogdanović, M. Eracleous, \& S. Sigurdsson, Astrophys. J. 697, 288 (2008); M. Dotti, et al., Mon. Not. R. Astron. Soc. 398, 73 (2009); T. A. Boroson, \& T. R. Lauer, Nature 258, 307 (2009); Astrophys. J, in press (2009) arXiv:0906.0020; G. A. Shields, et al., Astrophys. J. Lett., submitted (2009) arXiv:0907.3470.

[25] N. Turner, et al., Astrophys. J. 593, 992, 2003; 624, 267, (2005); S. Hirose, J. H. Krolik, \& O. Blaes, Astrophys. J. 691, 16, (2009).

[26] K. Ohsuga, et al., Pub. Astron. Soc. Jap. 61, L7, (2009).

[27] K. Hayasaki, Pub. Astron. Soc. Jap. 61, 65, (2009).

[28] http://www.sdss.org/

[29] http://www.sdss3.org/; the combination of SDSS and SDSS-III will provide two spectra of $\sim 10^{4}$ quasars at $z>2.1$, separated by $\sim 4$ observer years.

[30] http://www.lsst.org/lsst/

[31] http://www.astro.caltech.edu/ptf/. The quoted numbers are from E. Ofek, private communication (2009).

[32] C. Wolf, et al., Astron. \& Astrophys. 421, 913 (2004).

[33] D. Maoz, private communication (2009).

[34] S. Kaspi, et al., Astrophys. J. 659, 997 (2007); I. Botti, et al., arXiv:0805.4664 (2008).

[35] Wilhite, B. C., et al., Astrophys. J. 633, 638 (2005); ibid 641, 78 (2006); Lundgren, B. F. et al., Astrophys. J. 656, 73 (2007); D. E. Vanden Berk, et al., Astrophys. J. 601, 692 (2004); R. Chornock, et al., Astrophys. J., submitted (2009) arXiv:0906.0849.

[36] Wilhite, B. C., et al., Mon. Not. R. Astron. Soc. 383, 1232 (2008).

[37] B. M. Peterson, K. T. Korista, \& S. A. Cota, Astrophys. J. 312, L1 (1987); S. G. Sergeev, et al., Astrophys. J. 576, 660 (2002).

[38] Disk emission could also lead to a double peaked line profile for a single $\mathrm{BH}$ but with no orbital variability (e.g., M. Eracleous, et al., Astrophys. J. 490, 216 (1997)). 